Research Article

\title{
A Holling Type II Discrete Switching Host-Parasitoid System with a Nonlinear Threshold Policy for Integrated Pest Management
}

\author{
Mengqi He $\mathbb{D}^{\mathbb{D}},{ }^{1}$ Sanyi Tang $\mathbb{D}^{1},{ }^{1}$ and Robert A. Cheke $\mathbb{D}^{2}$ \\ ${ }^{1}$ School of Mathematics and Information Science, Shaanxi Normal University, Xi'an 710062, China \\ ${ }^{2}$ Natural Resources Institute, University of Greenwich at Medway, Central Avenue, Chatham Maritime, Chatham, Kent, \\ Gillingham ME4 4TB, UK
}

Correspondence should be addressed to Mengqi He; hemq@snnu.edu.cn and Sanyi Tang; sytang@snnu.edu.cn

Received 31 January 2020; Revised 16 April 2020; Accepted 11 May 2020; Published 9 June 2020

Academic Editor: Zhengqiu Zhang

Copyright (c) 2020 Mengqi He et al. This is an open access article distributed under the Creative Commons Attribution License, which permits unrestricted use, distribution, and reproduction in any medium, provided the original work is properly cited.

A nonlinear discrete switching host-parasitoid model with Holling type II functional response function, in which the switch is guided by an economic threshold (ET), is proposed. Thus, if the weighted density of two generations of the host population increases and exceeds the ET, then integrated pest management (IPM) measures are enacted, i.e., biological and chemical measures are implemented together, assuming that the chemical immediately precedes the biological inputs to avoid pesticideinduced deaths of the natural enemies. First, the existence and local stability of the equilibria of two subsystems were studied, and the existence and coexistence of several types of equilibria of a nonlinear switching system were analysed. Next, the nonlinear switching system was investigated by numerical simulation, showing that the system exhibits quite complex dynamic behaviour. A two-dimensional bifurcation diagram revealed the existence and coexistence regions of different types of equilibria including regular and virtual equilibria. Moreover, period-adding bifurcations in two-dimensional parameter spaces were found. Onedimensional bifurcation diagrams revealed that the system has periodic, quasiperiodic, and chaotic solutions, Neimark-Sacker bifurcation, multiple coexisting attractors, period-doubling bifurcations, period-halving bifurcations, and so on. Finally, the initial densities of hosts and parasitoids associated with host outbreaks and their biological implications are discussed.

\section{Introduction}

Pest control is important in subjects such as agriculture, fisheries, and ecology [1]. Pest outbreaks often cause huge economic losses and a series of ecological problems. For example, the outbreak of the fall armyworm moth Spodoptera frugiperda in 2019 has caused considerable economic losses in many areas of southern China [2]. Rational control of pests avoids indiscriminate spraying of pesticides, as long-term pesticide usage will not only induce resistance but also cause serious environmental pollution. In order to control pests more effectively and to consider the impact of control measures on the environment, the integrated pest management (IPM) strategy was proposed [3-6], which requires the combination of chemical control and biological control. There are some important concepts in IPM, including the economic injury level (EIL) [7-9], economic threshold (ET) [8-10], and threshold policy control (TPC) [11-13]. The purpose of IPM is not to eliminate pests completely, but to control them at levels below the EIL. Thus, the classical method is to maintain the density of a pest population below the EIL by spraying pesticides and releasing natural enemies and/or other measures such as cultural control once the density of the pest population reaches the ET.

Host-parasitoid models including IPM intervention with fixed and unfixed time pulse effects were first proposed and analysed by Tang et al. [8]. Moreover, Yang et al. [14] proposed and analysed a Holling type II host-parasitoid model with IPM intervention as a pulse control strategy, including models with fixed pulses and unfixed pulses. Wang and Zhang [15] studied the Holling type II host-parasitoid 
model with chemical control at a fixed time. In order to apply such a threshold policy control (TPC) to IPM measures, a switching system (or Filippov system) is proposed and studied. Such systems, based on the ordinary differential equation (ODE) model for the predator-prey interaction, have been investigated in recent publications (see $[12,13,16-18]$ for relevant references). In addition, Xiang and his co-authors [19-24] discussed the complex dynamic behaviour of discrete switching systems. However, in the above publications, the authors only considered the relationship between ET and the density of a pest population in generation $t$, that is, the threshold condition is linear or the switching surface is a straight line.

For a pest population with nonoverlapping generations, the best control threshold depends not only on the density of the current generation but also on the density of the next generation. This means that chemical control and biological control applied together (but with the chemical control immediately preceding the biological inputs to avoid pesticide-induced deaths of the natural enemies) should be guided by the densities of both generation $t$ and generation $t+1$. This means that the threshold condition is nonlinear, which we denote as a nonlinear switching system guided by the weighted density of two generations of the host population. To our knowledge, no previous research has been conducted on such a nonlinear discrete switching system.

The structure of this paper is as follows. In Section 2, a Holling type II host-parasitoid model with a nonlinear switching threshold strategy is proposed. In Section 3, we discuss the existence and local stability of the equilibria of the two subsystems. Numerical simulations including oneparameter bifurcation and two-parameter bifurcation are applied in Section 4 to reveal the complex and diverse dynamic behaviour of the nonlinear switching system. In Section 5, we study the importance and correlation of initial densities of both host and parasitoid populations in host outbreaks.

\section{Nonlinear Switching Host-Parasitoid Model}

A discrete host-parasitoid model with a Holling type II functional response function was numerically investigated by Tang and Chen [25], who showed that a discrete hostparasitoid model could generate much richer dynamics than those observed in continuous models. With $H$ the host and $P$ the parasitoid populations, this model is [25]

$$
\left\{\begin{array}{l}
H_{t+1}=H_{t} \exp \left[r\left(1-\frac{H_{t}}{K}\right)-\frac{a T P_{t}}{1+a T_{h} H_{t}}\right], \\
P_{t+1}=H_{t}\left[1-\exp \left(-\frac{a T P_{t}}{1+a T_{h} H_{t}}\right)\right]
\end{array}\right.
$$

where all parameters are positive, $r$ denotes the intrinsic growth rate of the host population in the absence of the parasitoid population, the carrying capacity is represented by $K, a$ describes the instantaneous search rate (the average encounters per host per unit of search time), assuming that the total time initially available for the search when the hosts are exposed to parasitoids is $T$, and $T_{h}$ is the handling time (the time between the host being encountered and the search for a host being resumed).

In order to take into account IPM strategies for controlling the pest population, Xiang et al. [21] considered that only when the density of generation $t$ of the host population exceeds the ET, is there implementation of biological control measures (natural enemy releases) together with chemical control measures (pesticide spraying, but see comment above on earlier deployment of pesticides), which means that the switching surface is a straight line.

But in reality, the optimal control threshold not only depends on the density of the current generation but also depends on the density of the next generation, which implies that the threshold ET will depend on both the host and parasitoid densities. The question is how to establish a mathematical model that uses the relationship between the population density of two adjacent generations and the threshold ET as the switching condition. In order to solve this problem, based on model (1), we consider a more generalized threshold control policy, i.e., once the weighted density of two generations of the host population exceeds the ET, the chemical control is formulated by assuming a proportional killing rate $p$ for the pest population, and the biological control is modelled by releasing a constant number of the natural enemy $\tau$, which obviously generates a nonlinear switching curve. This yields the following model:

$$
\left\{\begin{array}{c}
H_{t+1}=(1-p) H_{t} \exp \left[r\left(1-\frac{H_{t}}{K}\right)-\frac{a T P_{t}}{1+a T_{h} H_{t}}\right] \\
P_{t+1}=H_{t}\left[1-\exp \left(-\frac{a T P_{t}}{1+a T_{h} H_{t}}\right)\right]+\tau,
\end{array}\right\} \varepsilon H_{t}+(1-\varepsilon) H_{t+1} \geq \mathrm{ET},
$$

where $0 \leq p<1, \tau \geq 0,0 \leq \varepsilon \leq 1$. In order to control the pest, we assume that if $\tau>0$ and $p=0$, only biological control is adopted by the release of a sufficient number of the parasitoid to prevent the weighted host population exceeding ET, whereas if $0<p<1$ and $\tau=0$, chemical control is adopted to ensure that the weighted host population will be decreased once it reaches the ET. Note that if $\varepsilon=1$, then this special case was investigated by Xiang et al. [21]. 
Therefore, combining model (2) (called a control system) with IPM when the weighted density of two generations of the host population exceeds the ET and model (1) (called a free system) without control measures when the weighted density of two generations of the host population falls below the ET, we have the following nonlinear switching system:

$$
\left\{\begin{array}{l}
H_{t+1}=H_{t} \exp \left[r\left(1-\frac{H_{t}}{K}\right)-\frac{a T P_{t}}{1+a T_{h} H_{t}}\right] \\
P_{t+1}=H_{t}\left[1-\exp \left(-\frac{a T P_{t}}{1+a T_{h} H_{t}}\right)\right], \\
\varepsilon H_{t}+(1-\varepsilon) H_{t+1}<\mathrm{ET} ; \\
H_{t+1}=(1-p) H_{t} \exp \left[r\left(1-\frac{H_{t}}{K}\right)-\frac{a T P_{t}}{1+a T_{h} H_{t}}\right], \\
P_{t+1}=H_{t}\left[1-\exp \left(-\frac{a T P_{t}}{1+a T_{h} H_{t}}\right)\right]+\tau,
\end{array}\right\} \varepsilon H_{t}+(1-\varepsilon) H_{t+1} \geq \mathrm{ET} .
$$

Obviously, model (3) represents a switching system controlled by a nonlinear threshold policy and it differs from previous studies of a differential switching system in that the model switching surface is nonlinear. Note that the switching threshold between two subsystems depends not only on the density of current generation but also on the next generation, which results in a complex switching curve and could generate new dynamics related to controlling of host population. Moreover, once the weighted parameter $\varepsilon=0$ or 1 , then the switching curve reduces to the straight line, which has been studied in literatures [20,21].

\section{Stability Analysis of Two Subsystems}

In order to reveal the dynamics of the whole switching system (3), we first investigate the existence and stability of various equilibria of the control and free systems, providing results that are useful for studying the dynamical behaviour of system (3). For convenience, we denote $\varphi(Z)=\varepsilon H_{t}+$ $(1-\varepsilon) H_{t+1}-$ ET with vector $Z=\left(H_{t}, P_{t}\right)^{T}$, and

$$
\begin{aligned}
S_{G_{1}}(Z)= & {\left[H_{t} \exp \left(r\left(1-\frac{H_{t}}{K}\right)-\frac{a T P_{t}}{1+a T_{h} H_{t}}\right), H_{t}\left(1-\exp \left(-\frac{a T P_{t}}{1+a T_{h} H_{t}}\right)\right)\right]^{T}, } \\
S_{G_{2}}(Z)= & {\left[(1-p) H_{t} \exp \left(r\left(1-\frac{H_{t}}{K}\right)-\frac{a T P_{t}}{1+a T_{h} H_{t}}\right),\right.} \\
& \left.H_{t}\left(1-\exp \left(-\frac{a T P_{t}}{1+a T_{h} H_{t}}\right)\right)+\tau\right]^{T} .
\end{aligned}
$$

Then, model (3) can be rewritten as the following switching system:

$$
Z_{t+1}= \begin{cases}S_{G_{1}}(Z), & Z \in G_{1}, \\ S_{G_{2}}(Z), & Z \in G_{2},\end{cases}
$$

where

$$
\begin{aligned}
& G_{1}=\left\{Z \in R_{+}^{2} \mid \varphi(Z)<0, H_{t} \geq 0, P_{t} \geq 0\right\}, \\
& G_{2}=\left\{Z \in R_{+}^{2} \mid \varphi(Z) \geq 0, H_{t} \geq 0, P_{t} \geq 0\right\} .
\end{aligned}
$$

From now on, we call switching system (3) defined in region $G_{1}$ as subsystem $S_{G_{1}}$ (i.e., system (1)) and that defined in region $G_{2}$ as subsystem $S_{G_{2}}$ (i.e., system (2)).

Switching systems have different types of equilibria, and these equilibria play important roles in pest control. In order to show the existence of equilibria of switching system (4), we need the definitions of the regular equilibrium and the virtual equilibrium about switching system (4). 
Definition 1. A point $\mathrm{Z}^{*}=\left(H^{*}, P^{*}\right)$ is called a regular equilibrium of system (4) if $Z^{*}$ is an equilibrium of subsystem $S_{G_{1}}\left(S_{G_{1}}\left(Z^{*}\right)=0\right)$ and $\varphi\left(H^{*}, P^{*}\right)<0$ or if $Z^{*}$ is an equilibrium of subsystem $S_{G_{2}}\left(S_{G_{2}}\left(Z^{*}\right)=0\right)$ and $\varphi\left(H^{*}, P^{*}\right) \geq 0$. These equilibria will be denoted by $E_{R}^{1}$ and $E_{R}^{2}$. Similarly, a point $Z^{*}$ is called a virtual equilibrium of system (4) if $Z^{*}$ is an equilibrium of subsystem $S_{G_{1}}$ and $\varphi\left(H^{*}, P^{*}\right) \geq 0$ or if $Z^{*}$ is an equilibrium of subsystem $S_{G_{2}}$ and $\varphi\left(H^{*}, P^{*}\right)<0$. These equilibria will be denoted by $E_{\mathrm{V}}^{1}$ and $E_{V}^{2}$.

3.1. Local Stability of Equilibria for Subsystem $S_{G_{1}}$. If $\varepsilon H_{t}+(1-\varepsilon) H_{t+1}<$ ET, then the dynamics of system (4) have been determined by subsystem $S_{G_{1}}$. The equilibrium $\left(H^{*}, P^{*}\right)$ of subsystem $S_{G_{1}}$ satisfies the following equations:

$$
\begin{aligned}
& H^{*}=H^{*} \exp \left[r\left(1-\frac{H^{*}}{K}\right)-\frac{a T P^{*}}{1+a T_{h} H^{*}}\right], \\
& P^{*}=H^{*}\left[1-\exp \left(-\frac{a T P^{*}}{1+a T_{h} H^{*}}\right)\right] .
\end{aligned}
$$

Obviously, subsystem $S_{G_{1}}$ has the trivial equilibrium $E_{01}=(0,0)$.

Proposition 1. The trivial equilibrium $E_{01}$ is an unstable saddle.

Proof. The stability of $(0,0)$ of subsystem $S_{G_{1}}$ is determined by the eigenvalues of the following Jacobian matrix:

$$
\mathscr{J}_{0}=\left(\begin{array}{cc}
e^{r} & 0 \\
0 & 0
\end{array}\right) \text {. }
$$

The eigenvalues of $\mathscr{J}_{0}$ are given by $\lambda_{1}=e^{r}$ and $\lambda_{2}=0$. Since the parameter $r$ is positive, $\left|\lambda_{1}\right|>1,\left|\lambda_{2}\right|<1$. Hence, the trivial equilibrium $E_{01}$ is an unstable saddle.

In addition, there exists the parasitoid-free equilibrium $E_{b}=(K, 0)$. It was shown by Kaitala et al. [26] that the boundary equilibrium is locally asymptotically stable when $|1-r|<1$ and $\left|a T K /\left(1+a T_{h} K\right)\right|<1$. Meanwhile, when $R_{0}>1$ and $T\left(R_{0}-1\right)>T_{h} R_{0} \ln R_{0}$, there exists the unique positive equilibrium $E_{1}^{*}=\left(H_{1}^{*}, P_{1}^{*}\right)$, where $E_{1}^{*}$ satisfies the following equations:

$$
\begin{aligned}
& H_{1}^{*}=\frac{R_{0} \ln R_{0}}{a\left(T\left(R_{0}-1\right)-T_{h} R_{0} \ln R_{0}\right)}, \\
& P_{1}^{*}=\frac{\left(R_{0}-1\right) \ln R_{0}}{a\left(T\left(R_{0}-1\right)-T_{h} R_{0} \ln R_{0}\right)} .
\end{aligned}
$$

Here, $R_{0}$ denotes the net growth rate of the host per generation with $R_{0}=\exp \left[r\left(1-\left(H_{1}^{*} / K\right)\right)\right]$ and reveals that the equilibrium $E_{1}^{*}=\left(H_{1}^{*}, P_{1}^{*}\right)$ cannot be solved analytically.
In the following, we address the local stability of the unique positive equilibrium $E_{1}^{*}=\left(H_{1}^{*}, P_{1}^{*}\right)$ of subsystem $S_{G_{1}}$.

Theorem 1. The unique positive equilibrium $E_{1}^{*}=\left(H_{1}^{*}, P_{1}^{*}\right)$ of subsystem $S_{G_{1}}$ is asymptotically stable provided that

$$
\max \left\{-1-\operatorname{tr} \mathscr{F}_{1},-1+\operatorname{tr} \mathscr{J}_{1}\right\}<\operatorname{det} \mathscr{F}_{1}<1
$$

where

$$
\mathscr{J}_{1}=\left.\left(\begin{array}{ll}
\frac{\partial F_{1}}{\partial H} & \frac{\partial F_{1}}{\partial P} \\
\frac{\partial G_{1}}{\partial H} & \frac{\partial G_{1}}{\partial H}
\end{array}\right)\right|_{\left(H_{1}^{*}, P_{1}^{*}\right)} .
$$

Proof. In order to discuss the local stability of the equilibrium, we need to calculate the Jacobian matrix at the equilibrium. Firstly, we can rewrite subsystem $S_{G_{1}}$ as follows:

$$
\left\{\begin{array}{l}
H_{t+1}=F_{1}\left(H_{t}, P_{t}\right), \\
P_{t+1}=G_{1}\left(H_{t}, P_{t}\right) .
\end{array}\right.
$$

Therefore, we have

$$
\mathscr{J}_{1}=\left.\left(\begin{array}{ll}
\frac{\partial F_{1}}{\partial H} & \frac{\partial F_{1}}{\partial P} \\
\frac{\partial G_{1}}{\partial H} & \frac{\partial G_{1}}{\partial P}
\end{array}\right)\right|_{\left(H_{1}^{*}, P_{1}^{*}\right)},
$$

where

$$
\begin{aligned}
\left.\frac{\partial F_{1}}{\partial H}\right|_{\left(H_{1}^{*}, P_{1}^{*}\right)}= & 1-\frac{r R_{0} M}{K}+\frac{a^{2} T T_{h}\left(R_{0}-1\right) R_{0} M^{2}}{\left(1+a T_{h} R_{0} M\right)^{2}}, \\
\left.\frac{\partial F_{1}}{\partial P}\right|_{\left(H_{1}^{*}, P_{1}^{*}\right)}= & -\frac{a T R_{0} M}{1+a T_{h} R_{0} M}, \\
\left.\frac{\partial G_{1}}{\partial H}\right|_{\left(H_{1}^{*}, P_{1}^{*}\right)}= & 1-\left[1+\frac{a^{2} T T_{h}\left(R_{0}-1\right) R_{0} M^{2}}{\left(1+a T_{h} R_{0} M\right)^{2}}\right] \\
& \cdot \exp \left(-\frac{a T\left(R_{0}-1\right) M}{1+a T_{h} R_{0} M}\right), \\
\left.\frac{\partial G_{1}}{\partial P}\right|_{\left(H_{1}^{*}, P_{1}^{*}\right)}= & \frac{a T R_{0} M}{1+a T_{h} R_{0} M} \exp \left(-\frac{a T\left(R_{0}-1\right) M}{1+a T_{h} R_{0} M}\right),
\end{aligned}
$$

with

$$
M=\frac{\ln R_{0}}{a\left(T\left(R_{0}-1\right)-T_{h} R_{0} \ln R_{0}\right)},
$$

and then we can obtain the characteristic equation about the Jacobian matrix $\mathscr{J}_{1}$ of subsystem $S_{G_{1}}$ :

$$
\mathscr{F}(\lambda)=\lambda^{2}-\left(\operatorname{tr} \mathscr{F}_{1}\right) \lambda+\operatorname{det} \mathscr{F}_{1} \text {, }
$$

where 


$$
\begin{aligned}
\operatorname{tr} \mathscr{F}_{1}= & 1-\frac{r R_{0} M}{K}+\frac{a^{2} T T_{h}\left(R_{0}-1\right) R_{0} M^{2}}{\left(1+a T_{h} R_{0} M\right)^{2}} \\
& +\frac{a T R_{0} M}{1+a T_{h} R_{0} M} \exp \left(-\frac{a T\left(R_{0}-1\right) M}{1+a T_{h} R_{0} M}\right) \\
\operatorname{det} \mathscr{F}_{1}= & {\left[1-\frac{r R_{0} M}{K} \exp \left(-\frac{a T\left(R_{0}-1\right) M}{1+a T_{h} R_{0} M}\right)\right] \frac{a T R_{0} M}{1+a T_{h} R_{0} M} . }
\end{aligned}
$$

Conditions (5) ensure that all the inequalities

$$
\mathscr{F}(1)>0, \mathscr{F}(-1)>0, \operatorname{det} \mathscr{F}_{1}<1,
$$

hold, that is, the modulus of all roots of equation (16) is less than 1 . By employing the Jury condition, we can derive that the equilibrium point $\left(H_{1}^{*}, P_{1}^{*}\right)$ of subsystem $S_{G_{1}}$ is local asymptotically stable. The proof is completed.

3.2. Local Stability of Equilibria for Subsystem $S_{G_{2}}$. If $\varepsilon H_{t}+(1-\varepsilon) H_{t+1} \geq \mathrm{ET}$, then the dynamical behaviour of system (4) has been determined by subsystem $S_{G_{2}}$. Similarly, we can discuss the existence and stability of equilibria of subsystem $S_{G_{2}}$. Obviously, $S_{G_{2}}$ has a host-free equilibrium $E_{b}^{\prime}=(0, \tau)$ if $\tau>0$. Moreover, if

$$
\begin{gathered}
R_{1}>\frac{\exp (\tau a T)}{1-p}, \\
T\left[\left((1-p) R_{1}-1\right)\right]>T_{h}(1-p) R_{1} \ln \left((1-p) R_{1}\right),
\end{gathered}
$$

then subsystem $S_{G_{2}}$ has a unique positive equilibrium $E_{2}^{*}=\left(H_{2}^{*}, P_{2}^{*}\right)$, where

$$
\begin{aligned}
H_{2}^{*} & =\frac{(1-p) R_{1}\left(\ln \left((1-p) R_{1}\right)-\tau a T\right)}{a\left[T(1-p) R_{1}-T-T_{h}(1-p) R_{1} \ln \left((1-p) R_{1}\right)\right]} \\
P_{2}^{*} & =\frac{\left((1-p) R_{1}-1\right)\left(\ln \left((1-p) R_{1}\right)-\tau a T\right)}{a\left[T(1-p) R_{1}-T-T_{h}(1-p) R_{1} \ln \left((1-p) R_{1}\right)\right]}+\tau .
\end{aligned}
$$

Similarly, $R_{1}$ represents the net growth rate of the host per generation with $R_{1}=\exp \left[r\left(1-\left(H_{2}^{*} / K\right)\right)\right]$. Similarly, the equilibrium $E_{2}^{*}=\left(H_{2}^{*}, P_{2}^{*}\right)$ cannot be analytically solved. In the following theorems, the local stability of the host-free equilibrium $(0, \tau)$ and the positive equilibrium $\left(H_{2}^{*}, P_{2}^{*}\right)$ of subsystem $S_{G_{2}}$ are proved.

Theorem 2. The host-free equilibrium $(0, \tau)$ of subsystem $S_{G_{2}}$ is asymptotically stable provided that

$$
\left|(1-p) e^{r-\tau a T}\right|<1
$$

Proof. The stability of $(0, \tau)$ of subsystem $S_{G_{2}}$ is determined by the eigenvalues of the following Jacobian matrix:

$$
\mathscr{J}=\left(\begin{array}{cc}
(1-p) e^{r-\tau a T} & 0 \\
1-e^{-\tau a T} & 0
\end{array}\right) .
$$

Accordingly, we find that the eigenvalues are $\lambda_{1}=(1-p) e^{r-\tau a T}, \lambda_{2}=0$. From this, $(0, \tau)$ is asymptotically stable only when $\left|(1-p) e^{r-\tau a T}\right|<1$. The proof is completed.

For the stability of interior equilibrium $E_{2}^{*}=\left(H_{2}^{*}, P_{2}^{*}\right)$, we denote the Jacobian matrix as follows:

$$
\mathscr{J}_{2}=\left.\left(\begin{array}{ll}
\frac{\partial F_{2}}{\partial H} & \frac{\partial F_{2}}{\partial P} \\
\frac{\partial G_{2}}{\partial H} & \frac{\partial G_{2}}{\partial P}
\end{array}\right)\right|_{\left(H_{2}^{*}, P_{2}^{*}\right)},
$$

with

$$
\begin{aligned}
\left.\frac{\partial F_{2}}{\partial H}\right|_{\left(H_{2}^{*}, P_{2}^{*}\right)} & =1-\frac{r(1-p) R_{1} Q}{K}+\frac{a^{2} T T_{h}(1-p) R_{1} Q\left[\left((1-p) R_{1}-1\right) Q+\tau\right]}{\left(1+a T_{h}(1-p) R_{1} Q\right)^{2}}, \\
\left.\frac{\partial F_{2}}{\partial P}\right|_{\left(H_{2}^{*}, P_{2}^{*}\right)} & =-\frac{a T(1-p) R_{1} Q}{1+a T_{h}(1-p) R_{1} Q}, \\
\left.\frac{\partial G_{2}}{\partial H}\right|_{\left(H_{2}^{*}, P_{2}^{*}\right)} & =1-\left[1+\frac{a^{2} T T_{h}(1-p) R_{1} Q\left[\left((1-p) R_{1}-1\right) Q+\tau\right]}{\left(1+a T_{h}(1-p) R_{1} Q\right)^{2}}\right] \exp \left(-\frac{a T\left[\left((1-p) R_{1}-1\right) Q+\tau\right]}{1+a T_{h}(1-p) R_{1} Q}\right), \\
\left.\frac{\partial G_{2}}{\partial P}\right|_{\left(H_{2}^{*}, P_{2}^{*}\right)} & \frac{a T(1-p) R_{1} Q}{1+a T_{h}(1-p) R_{1} Q} \exp \left(-\frac{a T\left[\left((1-p) R_{1}-1\right) Q+\tau\right]}{1+a T_{h}(1-p) R_{1} Q}\right) \\
Q & =\frac{\ln \left((1-p) R_{0}\right)-\tau a T}{a\left[T(1-p) R_{0}-T-T_{h}(1-p) R_{0} \ln \left((1-p) R_{0}\right)\right]} .
\end{aligned}
$$


Theorem 3. The unique positive equilibrium $E_{2}^{*}=\left(H_{2}^{*}, P_{2}^{*}\right)$ of subsystem $S_{G_{2}}$ is asymptotically stable if and only if

$$
\max \left\{-1-\operatorname{tr} \mathscr{J}_{2},-1+\operatorname{tr} \mathscr{J}_{2}\right\}<\operatorname{det} \mathscr{F}_{2}<1 .
$$

The detailed mathematical expressions of $\operatorname{tr} \mathscr{J}_{2}$ and $\operatorname{det} \mathscr{J}_{2}$ can be given as follows:

$\operatorname{tr} \mathscr{J}_{2}=1-\frac{r(1-p) R_{1} Q}{K}+\frac{a^{2} T T_{h}(1-p) R_{1} Q\left[\left((1-p) R_{1}-1\right) Q+\tau\right]}{\left(1+a T_{h}(1-p) R_{1} Q\right)^{2}}+\frac{a T(1-p) R_{1} Q}{1+a T_{h}(1-p) R_{1} Q} \exp \left(-\frac{a T\left[\left((1-p) R_{1}-1\right) Q+\tau\right]}{1+a T_{h}(1-p) R_{1} Q}\right)$

$\operatorname{det} \mathscr{F}_{2}=\left[1-\frac{r(1-p) R_{1} Q}{K} \exp \left(-\frac{a T\left[\left((1-p) R_{1}-1\right) Q+\tau\right]}{1+a T_{h}(1-p) R_{1} Q}\right)\right] \frac{a T(1-p) R_{1} Q}{1+a T_{h}(1-p) R_{1} Q}$

The proof of Theorem 3 is similar to Theorem 1, and so we omit it.

\section{Complex Dynamical Behaviour Analysis}

In contrast to two subsystems (1) and (2), model (4) characterizes the nonlinear switching threshold policy, and its dynamical behaviour may be more complex compared with results obtained in the literature [25]. The nonlinear interaction between the host and the parasitoid populations and the nonlinear switching threshold is very difficult or even impossible to analyse theoretically, so we will study the dynamical behaviour of system (4) through numerical investigations.

4.1. Equilibria Bifurcation for Nonlinear Switching System (4). According to Definition 1, nonlinear switching system (4) has different types of equilibria, and these equilibria play key roles in disease and pest control. For example, Xiao et al. [27] investigated the control of emerging infectious disease and Tang et al. [16] addressed pest control with an ET. In the following, we focus on how the system with a nonlinear switching threshold affects the distribution of regular and virtual equilibria.

By a simple calculation, we conclude that if $R_{0}>1$,

$$
\begin{aligned}
T\left(R_{0}-1\right) & >T_{h} R_{0} \ln R_{0}, \\
a & >\frac{R_{0} \ln R_{0}}{E T\left(T\left(R_{0}-1\right)-T_{h} R_{0} \ln R_{0}\right)},
\end{aligned}
$$

then there exists a unique regular equilibrium for subsystem $S_{G_{1}}$, that is, $E_{R}^{1}=\left(H_{1}^{*}, P_{1}^{*}\right)$. If $R_{0}>1$,

$$
\begin{aligned}
T\left(R_{0}-1\right) & >T_{h} R_{0} \ln R_{0}, \\
a & <\frac{R_{0} \ln R_{0}}{\operatorname{ET}\left(T\left(R_{0}-1\right)-T_{h} R_{0} \ln R_{0}\right)},
\end{aligned}
$$

then $E_{R}^{1}$ becomes a virtual equilibrium denoted by $E_{V}^{1}$.

Similarly, if

$$
\begin{aligned}
R_{1} & >\frac{\exp (\tau a T)}{1-p}, \\
T\left[\left((1-p) R_{1}-1\right)\right]> & T_{h}(1-p) R_{1} \ln \left((1-p) R_{1}\right), \\
a & <\frac{(1-\varepsilon p) R_{1}\left(\ln \left((1-p) R_{1}\right)-\tau a T\right)}{\operatorname{ET}\left[T(1-p) R_{1}-T-T_{h}(1-p) R_{1} \ln \left((1-p) R_{1}\right)\right]},
\end{aligned}
$$

then there exists a unique regular equilibrium for subsystem $S_{G_{2}}$, that is, $E_{R}^{2}=\left(H_{2}^{*}, P_{2}^{*}\right)$. Moreover, if

$$
\begin{aligned}
& R_{1}>\frac{\exp (\tau a T)}{1-p}, \\
& T\left[\left((1-p) R_{1}-1\right)\right]>T_{h}(1-p) R_{1} \ln \left((1-p) R_{1}\right), \\
& a<\frac{(1-\varepsilon p) R_{1}\left(\ln \left((1-p) R_{1}\right)-\tau a T\right)}{\operatorname{ET}\left[T(1-p) R_{1}-T-T_{h}(1-p) R_{1} \ln \left((1-p) R_{1}\right)\right]},
\end{aligned}
$$


then $E_{R}^{2}$ becomes a virtual equilibrium denoted by $E_{V}^{2}$.

Further, if $R_{0}>1$,

$$
\begin{aligned}
T\left(R_{0}-1\right) & >T_{h} R_{0} \ln R_{0}, \\
R_{1} & >\frac{\exp (\tau a T)}{1-p}, \\
T\left[\left((1-p) R_{1}-1\right)\right] & >T_{h}(1-p) R_{1} \ln \left((1-p) R_{1}\right),
\end{aligned}
$$

then the two regular equilibria $E_{R}^{1}$ and $E_{R}^{2}$ can coexist only when $(q \doteq 1-p)$ :

$$
\begin{gathered}
\frac{R_{0} \ln R_{0}}{\operatorname{ET}\left(T\left(R_{0}-1\right)-T_{h} R_{0} \ln R_{0}\right)}<a \\
<\frac{(1-\varepsilon p) R_{1}\left(\ln \left(q R_{1}\right)-\tau a T\right)}{\operatorname{ET}\left[T q R_{1}-T-T_{h} q R_{1} \ln \left(q R_{1}\right)\right]} .
\end{gathered}
$$

Finally, if $R_{0}>1$,

$$
\begin{aligned}
T\left(R_{0}-1\right) & >T_{h} R_{0} \ln R_{0}, \\
R_{1} & >\frac{\exp (\tau a T)}{1-p}, \\
T\left[\left((1-p) R_{1}-1\right)\right] & >T_{h}(1-p) R_{1} \ln \left((1-p) R_{1}\right),
\end{aligned}
$$

then the two regular equilibria $E_{V}^{1}$ and $E_{V}^{2}$ can coexist only when

$$
\begin{gathered}
\frac{(1-\varepsilon p) R_{1}\left(\ln \left(q R_{1}\right)-\tau a T\right)}{\operatorname{ET}\left[T q R_{1}-T-T_{h} q R_{1} \ln \left(q R_{1}\right)\right]}<a \\
<\frac{R_{0} \ln R_{0}}{\operatorname{ET}\left(T\left(R_{0}-1\right)-T_{h} R_{0} \ln R_{0}\right)} .
\end{gathered}
$$

The interesting question is how the types of the interior equilibria of model (4) change as the parameters vary. Theoretically, it is impossible to answer this question due to the positions related to the switching curve of all interior equilibria of the two subsystems which cannot be analytically determined. Therefore, in the following, we reveal the existence of regular and virtual equilibria and their coexistence by two-parameter bifurcation analyses.

We first choose $a$ and ET as bifurcation parameters and fix the other parameters as given in Figure 1. Letting $a$ increase from 0.001 to 0.1 and with ET increasing from 0.01 to 1 , the results show that the parameter space is divided into eight regions. The results shown in Figure 1 reveal that the variations of two parameters $a$ and ET can significantly affect the types of equilibria of system (4), which are crucial for pest control and host outbreaks. For example, when the instantaneous search rate $a \in[0.001,0.0038]$, there is no interior equilibrium in the two subsystems $S_{G_{1}}$ and $S_{G_{2}}$, as shown in region I. If the instantaneous search rate $a$ in $[0.0038,0.011]$, the parameter space can be divided into two regions: region II- 1 and region II-2. In region II-1, only $E_{V}^{2}$ exists, while in region II-2, only $E_{R}^{2}$ exists. In particular, if we let $a=0.008$, then according to region II-2 of Figure 1 with $\mathrm{ET}=0.6$, the unique interior equilibrium of subsystem $S_{G_{2}}$ is

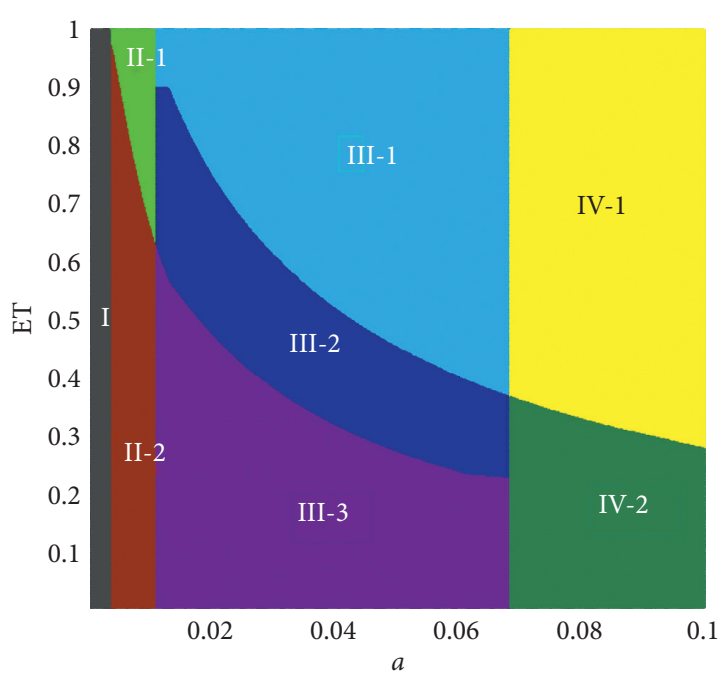

FIgURE 1: Bifurcation diagrams for the existence and coexistence of regular and virtual equilibria of system (4) with respect to parameters $a$ and ET. The different regions refer to different types of equilibria which are clarified in the text. The other parameters are fixed as follows: $r=3.5, p=0.1, T=100, T_{h}=1, \tau=0.4, K=1$, and $\varepsilon=0.3$.

regular ( $E_{R}^{2}$ here), but no interior equilibrium of subsystem $S_{G_{1}}$ exists. If ET further increases and goes beyond a certain threshold value $E T=0.76$, the regular equilibrium $E_{R}^{2}$ becomes a virtual equilibrium $E_{V}^{2}$ as shown in region II-1. If the instantaneous search rate $a$ is in $[0.011,0.069]$, the parameter space is divided into three regions which are regions III-1, III-2, and III-3. In region III- $1, E_{R}^{1}$ and $E_{V}^{2}$ coexist; in region III-2, $E_{V}^{1}$ and $E_{V}^{2}$ coexist; in region III-3, $E_{V}^{1}$ and $E_{R}^{2}$ coexist. When the instantaneous search rate $a$ increases and exceeds a certain threshold value $a=0.069$, that is, $a \in[0.069,0.1]$, then the parameter space is divided into two regions indicated as IV-1 and IV-2. In this case, the interior equilibrium of subsystem $S_{G_{2}}$ disappears, and only the interior equilibrium of subsystem $S_{G_{1}}$ exists. In region IV-1, only $E_{R}^{1}$ exists, while in region IV-2, only $E_{V}^{1}$ exists.

The stability and types of equilibria of free system are important for host control. For example, it is finally free of control if the equilibrium of the free system is globally stable. Therefore, in order to design the best control policies to prevent host outbreaks, one of the possible ways is to choose a desirable switching curve such that all equilibria of subsystem $S_{G_{1}}$ become regular and all equilibria of subsystem $S_{G_{2}}$ become virtual. Thus, the parameters $a$ and ET should be carefully chosen such that the interior equilibria of the two subsystems $S_{G_{1}}$ and $S_{G_{2}}$ are in region III-1. Similarly, we can address the effects of all other parameters on the regular and virtual equilibria of system (4).

4.2. Bifurcation Analysis about Key Parameters. In order to investigate the complex dynamic behaviour of system (4), we illustrate the one-dimensional parameter diagrams of the following parameters: the instantaneous search rate $a$, the intrinsic growth rate $r$, and the killing rate $p$. Moreover, we also give the two-dimensional parameter bifurcation plane 
of $r \times a$. The purpose of these numerical analyses is to reveal the complex dynamics and the types of attractors and their changes as the parameters change.

We first choose the instantaneous search rate $a$ as the bifurcation parameter and fix the other parameters as shown in Figure 2. It follows from Figure 2 that nonlinear switching system (4) has more complex and interesting dynamic behaviour as the instantaneous search rate increases. When $a$ increases from 0.03 to 0.06 , we can find periodic, quasiperiodic, and chaotic oscillations and a Neimark-Sacker bifurcation [28-30]. As the parameter $a$ increases from 0.03 to 0.033 , system (4) has a stable solution (see Figure 3(a) for details when $a=0.032$ ). When $a$ is further increased to 0.03305 , an attracting invariant curve generates a Neimark-Sacker bifurcation, which is the discrete-time analogue of a Hopf bifurcation (see Figures 3(b) and 3(c) for details). Moreover, the quasiperiodic attractor appears suddenly at $a=0.0345$ and $a=0.0455$, at which the quasiperiodic attractors in the phase plane give one and eight closed curves (see Figures 3(d) and 3(e)). However, a chaotic solution emerges abruptly at $a=0.055$ (see Figure 3(f) for details).

In addition, when we choose the intrinsic growth rate $r$ and the killing rate $p$ as the bifurcation parameters, system (4) also shows complex dynamic behaviour as shown in Figures 4-6. It follows from the bifurcation diagram with respect to parameter $r$ (see Figure 4) that there is perioddoubling bifurcation for a range of parameter values, that is, a solution with period-16 to period-32 by the period-doubling bifurcation and then the solution with period-32 to period-16 through the period-halving phenomena when $r \in[2.99,3.14]$. Moreover, we can also observe the coexistence of multiple attractors, for example, $r \in[2.63,2.66]$. The details will be discussed in the following section.

To investigate the effect of different parameter values on the periodicity of solutions for both host and parasitoid populations, according to published methods [31-34], a two-dimensional bifurcation diagram is developed in Figure 5 using the intrinsic growth rate $r$ and the instantaneous search rate $a$ as bifurcation parameters, in which the periods of period point cycles have been marked with different colors. With parameter increases, system (4) experiences period-adding bifurcation and there are many periodic windows embedded in the chaotic areas. The periodic solutions with different periods are plotted in different colors and marked by the corresponding numbers, in particular, the number 2 represents a period 2-point cycle, the number 14 represents period 14-point cycle, and the chaotic region has been marked when the number is equal or greater than 30 . The variations of complex parameter spaces related to the periods of solutions of system (4) reveal that small changes of key parameters could significantly influence the oscillation patterns of both host and parasitoid populations. Consequently, this could result in difficulties for successful pest control.

The bifurcation diagram with respect to parameter $p$ shown in Figure 6 reveals a phenomenon such that if the killing rate of the pesticides is too low or too large (e.g., $p \in[0.01,0.22]$ and $[0.81,0.98])$, the host population will have outbreaks. From the ecological point of view, if we choose a pesticide with low efficacy, i.e., $p \in[0.01,0.22]$, it may not be able to kill pests and eventually lead to outbreaks; if we choose a pesticide with high efficacy, i.e., $p \in[0.81,0.98]$, it may kill natural enemies while killing pests, but in this case, the pest population can still have an outbreak. All these results reveal that when using high-dose insecticides to kill hosts in large quantities, the parasitoid population will die in large numbers later due to the lack of hosts, which will eventually lead to the resurgence of the host population. Moreover, the main results shown in Figure 6 clearly reveal how to choose the appropriate killing rate to make the whole system stabilize at the attractor of subsystem $S_{G_{1}}$. In particular, if we choose the killing rate $p \in[0.23,0.80]$, then the pest population can stabilize in subsystem $S_{G_{1}}$ so as to prevent the outbreak of pests.

\section{Initial Sensitivities and Coexistence of Multiple Attractors}

It is well known that the initial densities of host and parasitoid populations will have a significant impact on the dynamic behaviour of the system, so they are key to biological control, and in this section, we will investigate how they affect outbreak patterns or final states of the host population.

5.1. Initial Sensitivities. In order to analyse the relationship between initial densities of populations and host outbreak patterns, Figure 7 illustrates the effect of initial densities on the outbreak frequencies related to the nonlinear switching curve. In Figure 7(a), the initial densities of the host and parasitoid populations are $(0.4,0.5)$, respectively, and the numerical results indicate that the density of the host population never reaches the switching surface, which shows that the solutions initiating from $(0.4,0.5)$ are free from IPM control measures. If we choose the initial densities as $(0.4,0.65)$ and $(0.5,0.56)$, the simulation results indicate that the host population will not have an outbreak after applying one or two IPM strategies (see Figures 7(b) and 7(c) for details). However, if we set the initial densities to be $(0.5,0.6)$, in order to control the weighted density of the host population below ET, the IPM measures should be implemented multiple times, as shown in Figure $7(\mathrm{~d})$.

In order to reveal the effects of initial values of both host and parasitoid populations on the outbreak patterns of the host population in more detail, the basin of attraction of the host outbreak frequencies with respect to initial densities is shown in Figure 8, and the regions I, II, III, IV, and V with different colors clearly clarify the initial sensitivities. An outbreak of the weighted host population could not occur in region I, where the system is always stabilized at the attractor of subsystem $S_{G_{1}}$. In regions II, III, and IV, the weighted density of two generations of the host density can be lower than the given threshold ET after implementing the IPM strategy one, two, and three times, while the weighted host population will have outbreaks several times if the initial densities lie in region $\mathrm{V}$ before the system stabilizes in 


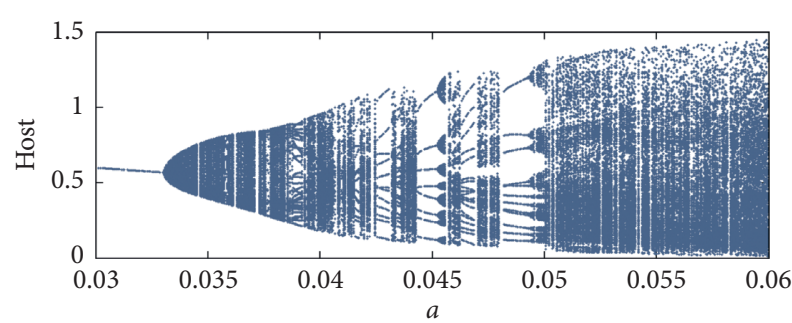

(a)

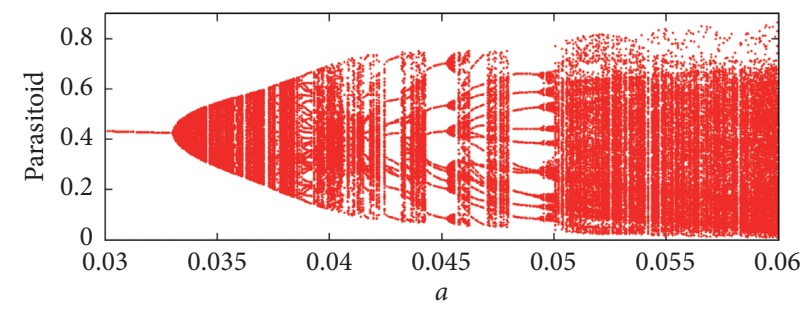

(b)

Figure 2: Bifurcation diagrams of system (4) with respect to parameter $a$. The other parameters are fixed as follows: $r=3.2, p=0.5, T=100, T_{h}=1, \tau=0.1, K=1, E T=0.8$, and $\varepsilon=0.5$.

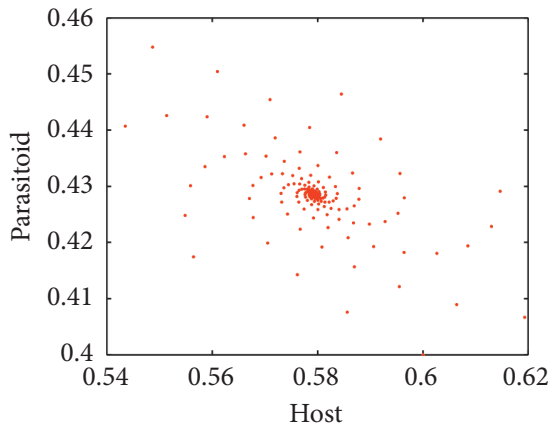

(a)

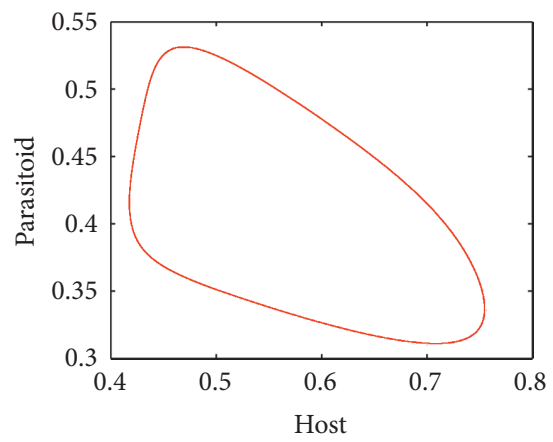

(d)

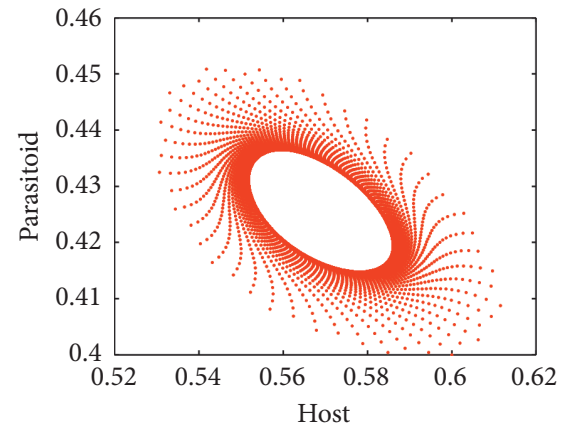

(b)

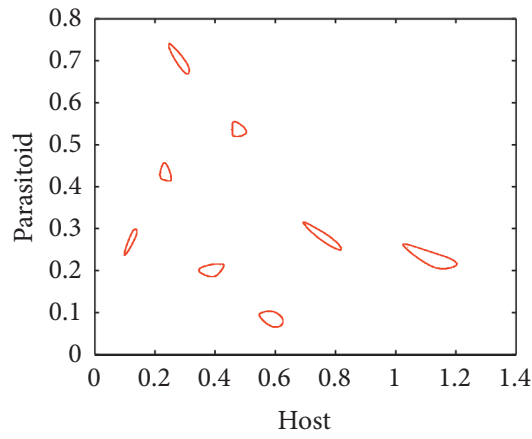

(e)

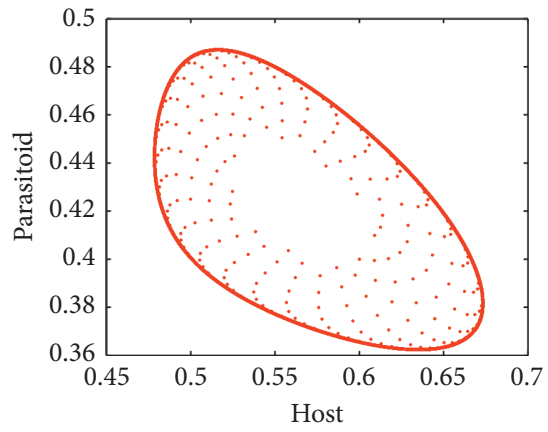

(c)

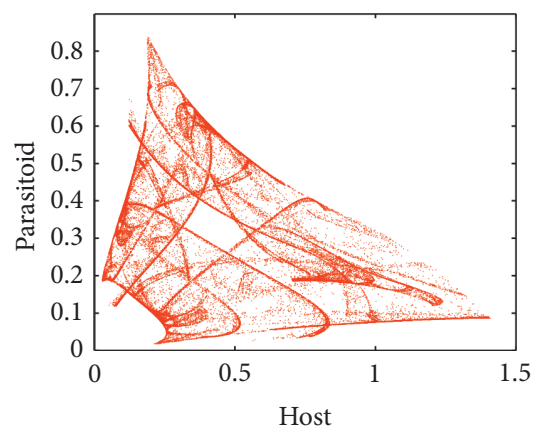

(f)

Figure 3: Phase trajectories of system (4) with different $a$ and initial values. (a) $a=0.032$. (b) $a=0.03305$. (c) $a=0.0335$. (d) $a=0.0345$. (e) $a=0.0455$. (f) $a=0.055$. The initial values $\left(H_{0}, P_{0}\right)$ in (a)-(c) are $(0.6,0.4)$ and in (d) $-(\mathrm{f})$ are $(0.7,0.31)$. The other parameters are fixed as follows: $r=3.2, p=0.5, T=100, T_{h}=1, \tau=0.1, K=1, E T=0.8$, and $\varepsilon=0.5$.

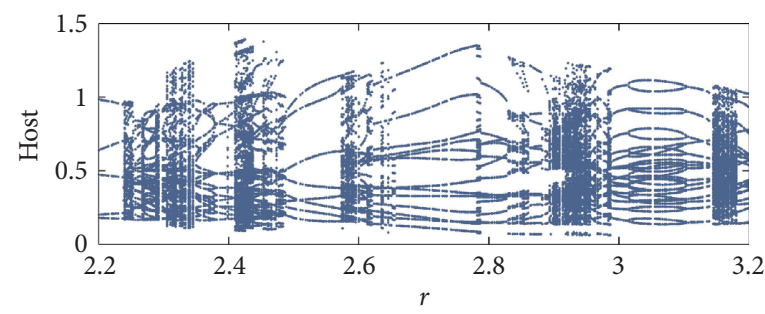

(a)

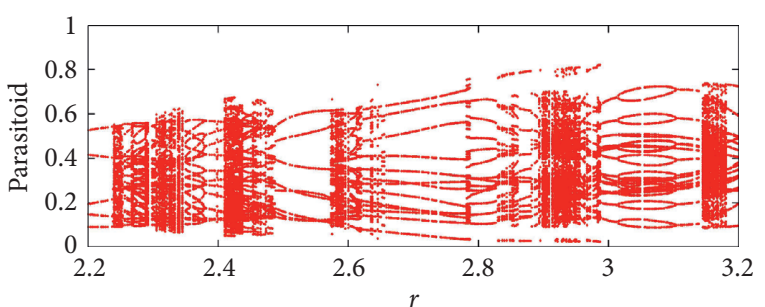

(b)

Figure 4: Bifurcation diagrams of system (4) with respect to parameter $r$. The other parameters are fixed as follows: $a=0.041, T=100, T_{h}=1, p=0.5, \tau=0.1, K=1, \mathrm{ET}=0.8$, and $\varepsilon=0.6$. 


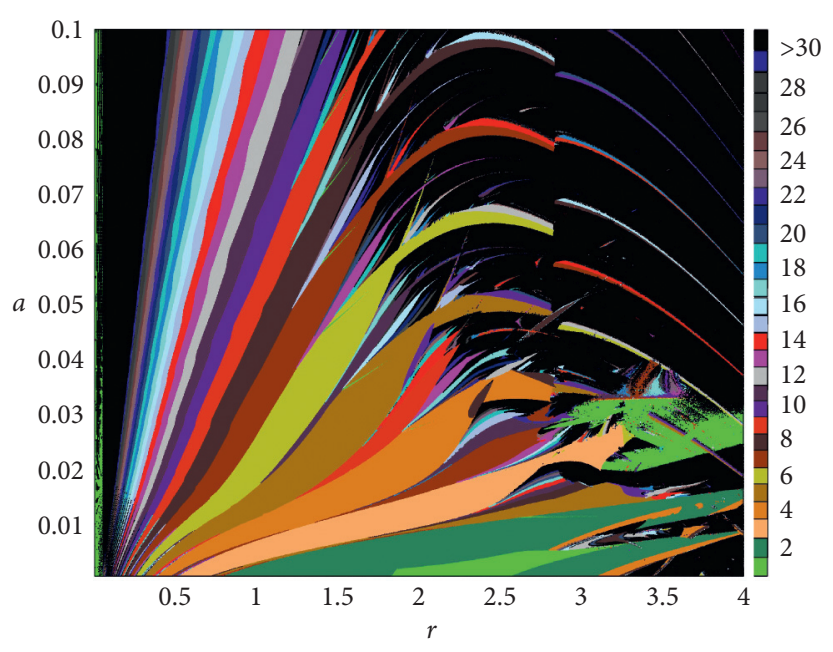

Figure 5: Periods of period point cycles of system (4) with respect to parameters $r$ and $a$, where different color intensities represent different periodic values. The other parameters are fixed as follows: $p=0.4, T=100, T_{h}=1, \tau=0.6, K=1, \mathrm{ET}=0.8$, and $\varepsilon=0.6$.

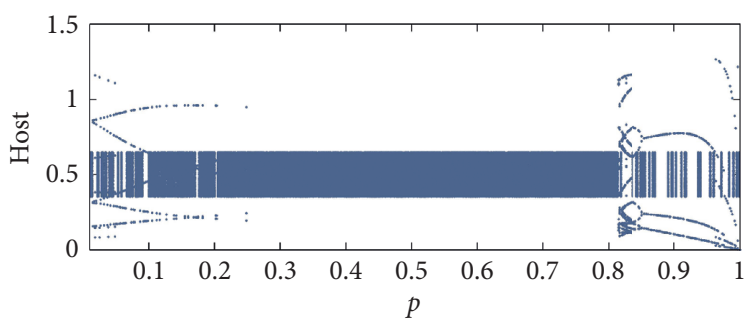

(a)

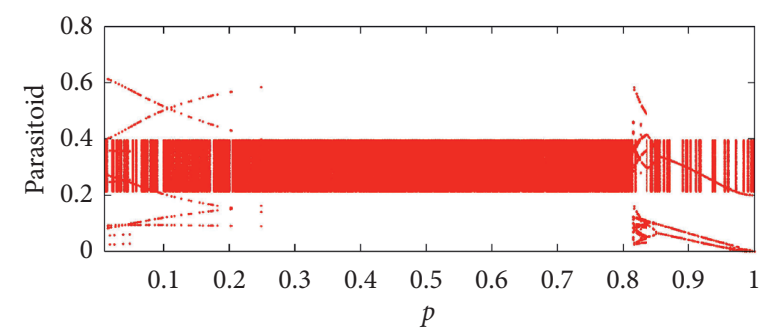

(b)

Figure 6: Bifurcation diagrams of system (4) with respect to parameter $p$. The other parameters are fixed as follows: $r=1.9, a=0.033, T=100, T_{h}=1, \tau=0.2, K=1, \mathrm{ET}=0.8$, and $\varepsilon=0.6$.

subsystem $S_{G_{1}}$. All these results confirm that the initial densities of both populations are crucial for the outbreak patterns and frequencies of host population.

5.2. Coexistence of Multiple Attractors. The initial values not only influence the host outbreak frequencies but can also affect the host final stable states, i.e., multiple attractors could coexist. It follows from the bifurcation analyses that multiple attractors can coexist for a wide range of parameters (see Figure 4 as an example). To further confirm this and address their biological implications, we set all parameters in Figure 9 and choose different initial densities to depict some typical trajectories. Especially, three host outbreak attractors coexist when $r=2.288$, as shown in Figure 9 , which show different periods. If we set the initial densities to be $(0.4,0.3)$, then the solution of system (4) approaches the periodic attractor with period 9 as shown in Figure $9(\mathrm{a})$. If we choose the initial values as $(0.4,0.1)$, then the solution of system (4) is a periodic attractor of period 14 (see Figure 9(b)). Moreover, when the initial value is $(0.5,0.3)$, the third attractor becomes quite complex (Figure 9(c)).

In order to illustrate the initial sensitivities more specifically, the basin of attraction with respect to three different host outbreak solutions of coexistence is shown in Figure 10. A basin of attraction exists for three attractors: the blue, magenta, and green regions. These are related to the periodic solutions which are shown in Figures 9(a)-9(c). We can also see that the curve $\varepsilon H_{t}+(1-\varepsilon) H_{t+1}=$ ET divides the attraction regions into two distinct areas, each of which has a significant difference in dynamic behaviour. Moreover, the sensitivity and dependence of initial values are very obvious, and the final attractors of host and parasitoid populations depend on their initial densities. Note that all these results are confirmed by basins of attraction of initial densities.

\section{Discussion}

In this paper, we discussed a nonlinear switching discrete host-parasitoid model with Holling type II functional response function, where the switching strategies and control measures are guided by the weighted density of two generations of the pest population. The important innovation of the model lies in the threshold control strategy. Here, we consider the weighted density of two generations of the pest population as the threshold to judge whether the control strategy is implemented or not, which not only brings new challenges in theory but also makes pest control more operable. The most interesting theoretical analyses are to 


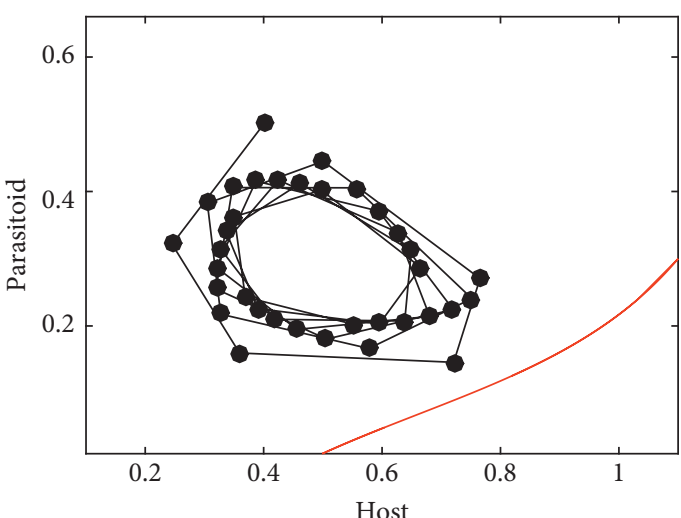

(a)

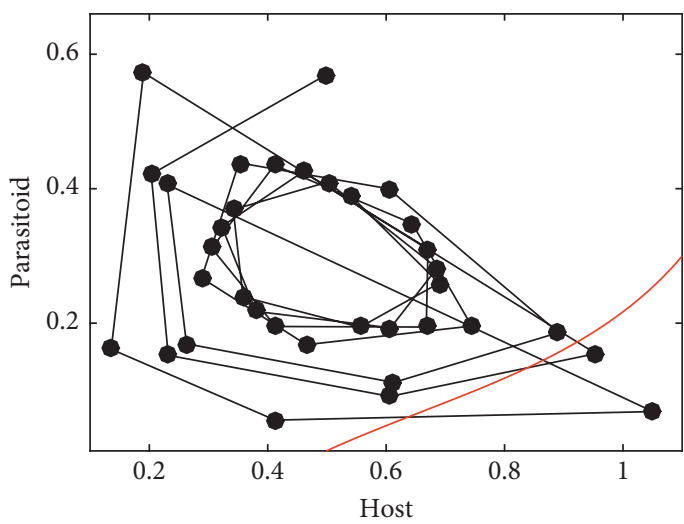

(c)

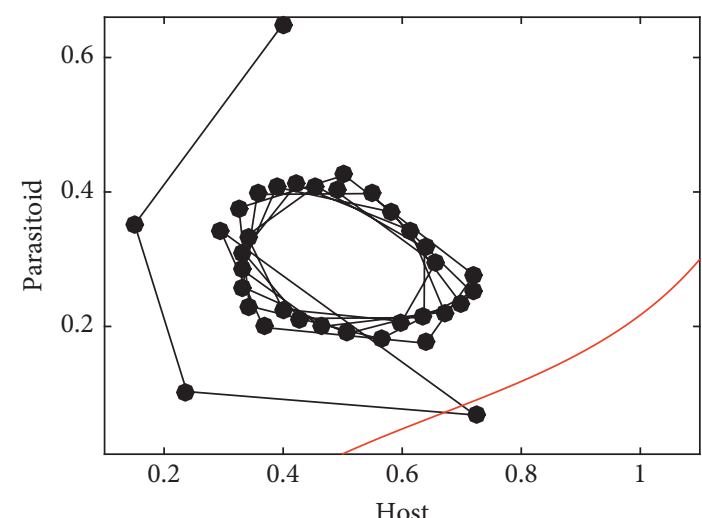

(b)

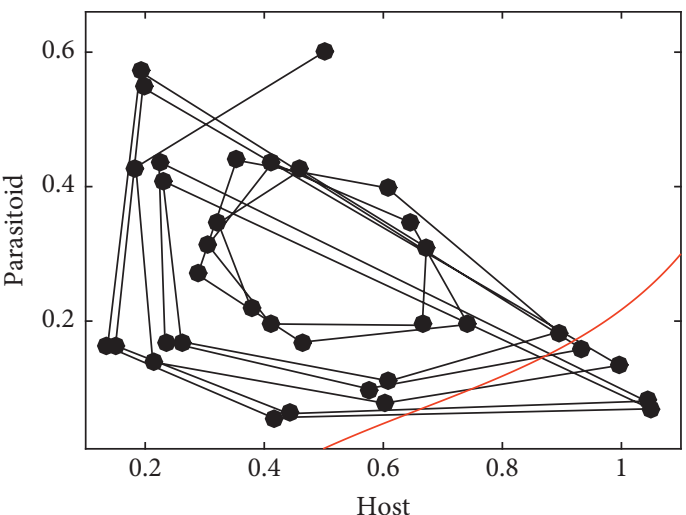

(d)

FiguRE 7: Illustrating the switching effects of initial densities of the host and parasitoid populations of system (4) related to the switching curve. The parameters are fixed as follows: $r=1.9, a=0.033, T=100, T_{h}=1, \tau=0.2, K=1, \mathrm{ET}=0.8$, and $\varepsilon=0.6$. The initial values $\left(H_{0}, P_{0}\right)$ in $(\mathrm{a})-(\mathrm{d})$ are $(0.4,0.5),(0.4,0.65),(0.5,0.56)$, and $(0.5,0.6)$. The red lines in each subplot represent the switching curves.

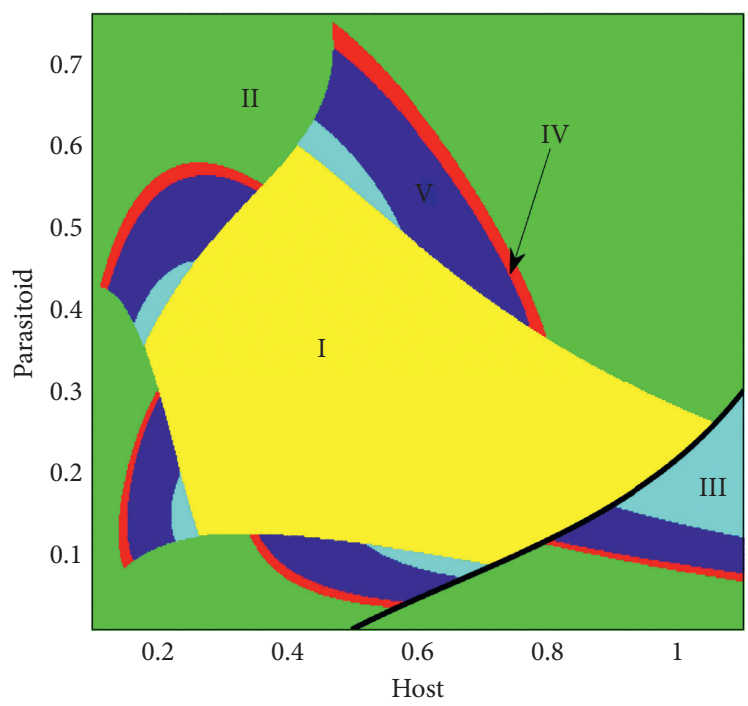

Figure 8: Dependence of the host outbreak frequencies on the initial values $\left(H_{0}, P_{0}\right)$ of system (4). The parameters are fixed as follows: $r=1.9, p=0.7, a=0.033, T=100, T_{h}=1, \tau=0.2, K=1, \mathrm{ET}=0.8$, and $\varepsilon=0.6$.

determine the types of all possible equilibria of the whole switching system and their stabilities because if the real equilibrium of the free subsystem is stable for the whole switching system, then the purpose of pest control can be realized easily. Further, the sensitivity analyses related to the key parameters and initial densities of both host and 


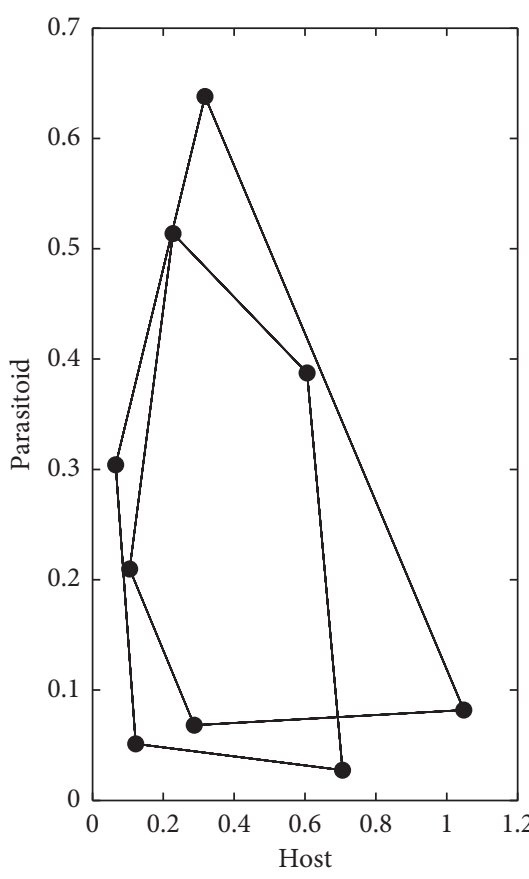

(a)

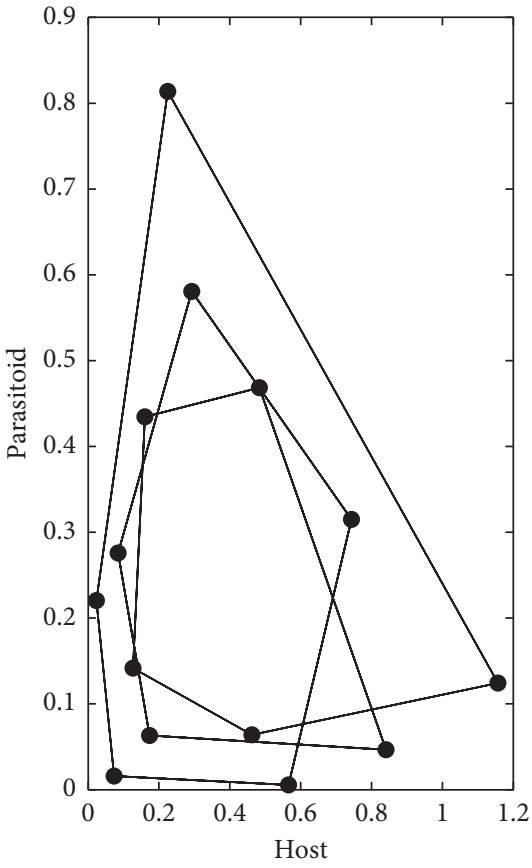

(b)

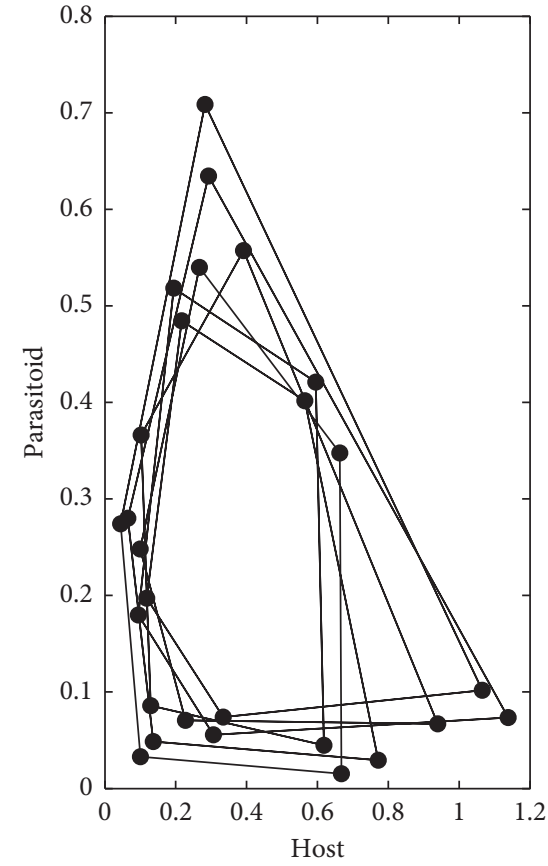

(c)

FIgURE 9: Three coexisting attractors of system (4) with different initial values. The parameters are fixed as follows: $r=2.288, a=0.05, T=100, T_{h}=1, p=0.5, \tau=0.3, K=1, \mathrm{ET}=0.75$, and $\varepsilon=0.6$. (a) $\left(H_{0}, P_{0}\right)=(0.4,0.3) .(\mathrm{b})\left(H_{0}, P_{0}\right)=(0.4,0.1) .(\mathrm{c})$ $\left(H_{0}, P_{0}\right)=(0.5,0.3)$.

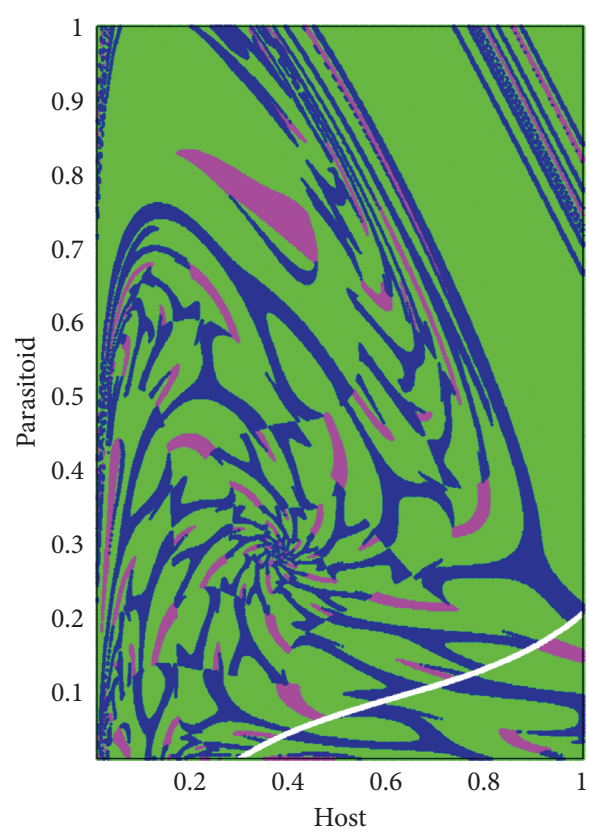

(a)

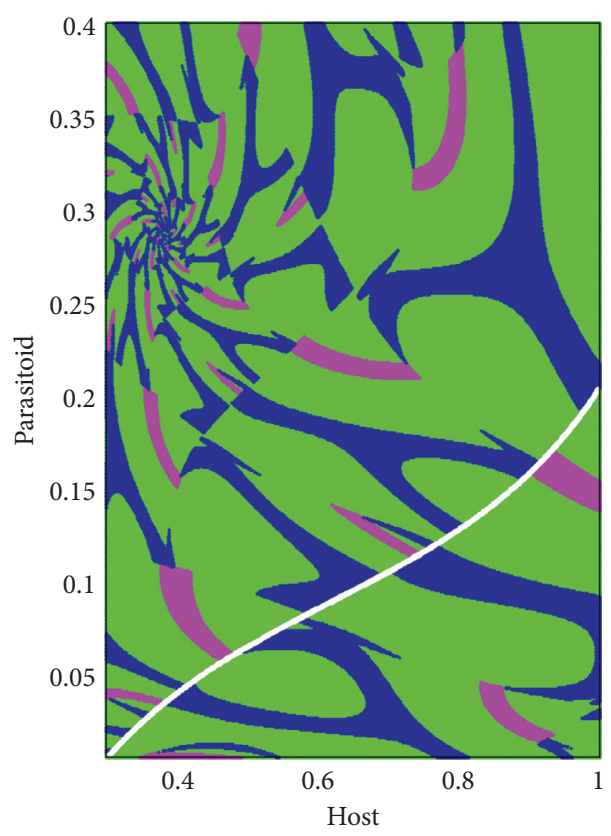

(b)

FIGURE 10: Basins of attraction of three coexisting attractors of system (4). The range of the left one is $0.01 \leq H_{0} \leq 1,0.01 \leq P_{0} \leq 1$. The blue, magenta, and green regions are attracted to the attractors shown in Figure 9 from left to right. The right one is an enlargement of the basins of attraction with range $0.3 \leq H_{0} \leq 1,0.01 \leq P_{0} \leq 0.4$. The parameters are fixed as follows: $r=2.288, a=0.05, T=100, T_{h}=1$, $p=0.5, \tau=0.3, K=1, \mathrm{ET}=0.75$, and $\varepsilon=0.6$. The white lines in each subplot represent the switching curves. 
parasitoid populations can help us to design suitable control measures.

We first investigated the existence and local stability of equilibria of two subsystems and briefly analysed the existence and coexistence of the equilibria of the whole nonlinear switching system. We used the $a \times$ ET twoparameter bifurcation diagram to reveal the distribution regions of different types of equilibria including regular and virtual equilibria, as shown in Figure 1. From the perspective of pest control, the parameters $a$ and ET should be carefully chosen such that the interior equilibria of the two subsystems $S_{G_{1}}$ and $S_{G_{2}}$ are in region III-1. Moreover, we provided the $r \times a$ two-parameter bifurcation diagram that reveals the existence of period-adding bifurcation in this nonlinear switching system, as shown in Figure 5. This suggests that we should choose appropriate parameters to avoid the solution of system (4) falling into the chaotic region for successful implementation of an IPM strategy.

On the other hand, the one-parameter bifurcation diagrams which were derived from system (4) reveal the complex dynamical behaviour. For example, the bifurcation diagram of the instantaneous search rate $a$ shows that system (4) may have very complex dynamical behaviour such as periodic, quasiperiodic, and chaotic solutions and a Neimark-Sacker bifurcation (Figures 2 and 3). The bifurcation diagram of the intrinsic growth rate $r$ illustrates that system (4) may have multiple coexisting attractors, period-doubling bifurcation, and period-halving bifurcation (Figure 4). Moreover, it follows from the bifurcation diagram of the killing rate $p$ that the host population can stabilize in subsystem $S_{G_{1}}$ (Figure 6), which emphasises the importance of choosing the appropriate concentration of pesticide.

In addition, the relationship between initial densities and pest control was also studied. The results show that the initial densities of the host and parasitoid populations will affect the outcome of an IPM strategy, and the final stable states of the populations depend on their initial densities (Figures 7-10). In those figures, the basins of initial attractors and outbreak frequencies of the host population have been revealed, which clarify that the outbreak patterns of the host population could become markedly different as parameters vary and change.

Compared with the basic model and the main published results [21], we conclude that it is more flexible for long-term monitoring and successful control of the host population if we employ the weighted density of two generations of its population as the standard for deciding whether the control strategy is implemented or not. In particular, in a certain parameter space, the nonlinear switching curve can ensure that the stable region of the real equilibrium of the free subsystem increases, thus increasing the controllability of the host population. Moreover, the one-parameter bifurcation and two-parameter bifurcation analyses reveal that the dynamic behaviour of system (4) is more variable, including the appearance of Neimark-Sacker bifurcations and period-adding bifurcations. Obviously, the nonlinear switching curve divides the basin of attraction of outbreak frequencies and coexistence attractors of the host and parasite populations into two distinct patterns, as shown in Figures 8 and 10, respectively.

\section{Data Availability}

No data were used to support this study. In our study, there are only some numerical simulations to support our main result, and parameter values to support the result of this paper are included within the article.

\section{Conflicts of Interest}

The authors declare that there are no conflicts of interest regarding the publication of this article.

\section{Acknowledgments}

This study was supported by the National Natural Science Foundation of China (NSFC) (61772017 and 11631012) and by the Fundamental Research Funds for the Central Universities (GK201901008).

\section{References}

[1] F. J. J. A. Bianchi, C. J. H. Booij, and T. Tscharntke, "Sustainable pest regulation in agricultural landscapes: a review on landscape composition, biodiversity and natural pest control," Proceedings of the Royal Society B: Biological Sciences, vol. 273, no. 1595, pp. 1715-1727, 2006.

[2] X. X. Sun, C. X. Hu, H. R. Jia et al., "Case study on the first immigration of fall armyworm Spodoptera frugiperda invading into China," Journal of Integrative Agriculture, vol. 18, pp. 2-10, 2019.

[3] D. Chandler, A. S. Bailey, G. M. Tatchell, G. Davidson, J. Greaves, and W. P. Grant, "The development, regulation and use of biopesticides for integrated pest management," Philosophical Transactions of the Royal Society B: Biological Sciences, vol. 366, no. 1573, pp. 1987-1998, 2011.

[4] B. J. Jacobsen, N. K. Zidack, and B. J. Larson, "The role of bacillus-based biological control agents in integrated pest management systems: plant diseases," Phytopathology, vol. 94, no. 11, pp. 1272-1275, 2004.

[5] P. F. J. Wolf and J. A. Verreet, "An integrated pest management system in Germany for the control of fungal leaf diseases in sugar beet: the IPM sugar beet model," Plant Disease, vol. 86, no. 4, pp. 336-344, 2002.

[6] M. P. Parrella and V. P. Jones, "Development of integrated pest management strategies in floricultural crops," Bulletin of the Entomological Society of America, vol. 33, no. 1, pp. 28-34, 1987.

[7] L. P. Pedigo, S. H. Hutchins, and L. G. Higley, "Economic injury levels in theory and practice," Annual Review of Entomology, vol. 31, no. 1, pp. 341-368, 1986.

[8] S. Tang, Y. Xiao, and R. A. Cheke, "Multiple attractors of hostparasitoid models with integrated pest management strategies: eradication, persistence and outbreak," Theoretical Population Biology, vol. 73, no. 2, pp. 181-197, 2008.

[9] D. W. Onstad, "Calculation of economic-injury levels and economic thresholds for pest management," Journal of Economic Entomology, vol. 80, no. 2, pp. 297-303, 1987.

[10] H. C. Chiang, "General model of the economic threshold level of pest populations," Plant Protection Bulletin, vol. 27, pp. 71-73, 1979. 
[11] Y. Yang and X. F. Liao, "Filippov Hindmarsh-Rose neuronal model with threshold policy control," IEEE Transactions on Neural Networks and Learning Systems, vol. 30, no. 1, pp. 306-311, 2018.

[12] S. Tang, G. Tang, and W. Qin, "Codimension-1 sliding bifurcations of a filippov pest growth model with threshold policy," International Journal of Bifurcation and Chaos, vol. 24, no. 10, p. 1450122, 2014.

[13] W. Qin, X. Tan, M. Tosato, and X. Liu, "Threshold control strategy for a non-smooth Filippov ecosystem with group defense," Applied Mathematics and Computation, vol. 362, p. 124532, 2019.

[14] J. Yang, S. Tang, and Y. Tan, "Complex dynamics and bifurcation analysis of host-parasitoid models with impulsive control strategy," Chaos, Solitons \& Fractals, vol. 91, pp. 522-532, 2016.

[15] T. Wang and Y. T. Zhang, "Chemical control for host-parasitoid model within the parasitism season and its complex dynamics," Discrete Dynamics in Nature and Society, vol. 2016, Article ID 3989625, 14 pages, 2016.

[16] S. Tang, J. Liang, Y. Xiao, and R. A. Cheke, "Sliding bifurcations of Filippov two stage pest control models with economic thresholds," SIAM Journal on Applied Mathematics, vol. 72, no. 4, pp. 1061-1080, 2012.

[17] A. Wang and Y. Xiao, "A Filippov system describing media effects on the spread of infectious diseases," Nonlinear Analysis: Hybrid Systems, vol. 11, pp. 84-97, 2014.

[18] X. Zhang and S. Tang, "Existence of multiple sliding segments and bifurcation analysis of Filippov prey-predator model," Applied Mathematics and Computation, vol. 239, pp. 265-284, 2014.

[19] X. Hu, W. Qin, and M. Tosato, "Complexity dynamics and simulations in a discrete switching ecosystem induced by an intermittent threshold control strategy," Mathematical Biosciences and Engineering, vol. 17, no. 3, pp. 2164-2178, 2020.

[20] W. Qin, X. Tan, X. Shi, and C. Xiang, "IPM strategies to a discrete switching predator-prey model induced by a matefinding Allee effect," Journal of Biological Dynamics, vol. 13, no. 1, pp. 586-605, 2019.

[21] C. Xiang, Z. Xiang, S. Tang, and J. Wu, "Discrete switching host-parasitoid models with integrated pest control," International Journal of Bifurcation and Chaos, vol. 24, no. 9, Article ID 1450114, 2014.

[22] C. C. Xiang, Z. Y. Xiang, and Y. Yang, "Dynamic complexity of a switched host-parasitoid model with Beverton-Holt growth concerning integrated pest management," Journal of Applied Mathematics, vol. 2014, Article ID 501423, 10 pages, 2014.

[23] C. Xiang, S. Tang, R. A. Cheke, and W. Qin, "A locust phase change model with multiple switching states and random perturbation," International Journal of Bifurcation and Chaos, vol. 26, no. 13, Article ID 1630037, 2016.

[24] C. C. Xiang, Y. Yang, Z. Y. Xiang, and W. J Qin, "Numerical analysis of discrete switching prey-predator model for integrated pest management," Discrete Dynamics in Nature and Society, vol. 2016, Article ID 8627613, 11 pages, 2016.

[25] S. Tang and L. Chen, "Chaos in functional response hostparasitoid ecosystem models," Chaos, Solitons \& Fractals, vol. 13, no. 4, pp. 875-884, 2002.

[26] V. Kaitala, J. Ylikarjula, and M. Heino, "Dynamic complexities in host-parasitoid interaction," Journal of Theoretical Biology, vol. 197, no. 3, pp. 331-341, 1999.
[27] Y. Xiao, X. Xu, and S. Tang, "Sliding mode control of outbreaks of emerging infectious diseases," Bulletin of Mathematical Biology, vol. 74, no. 10, pp. 2403-2422, 2012.

[28] Z. He and X. Lai, "Bifurcation and chaotic behavior of a discrete-time predator-prey system," Nonlinear Analysis: Real World Applications, vol. 12, no. 1, pp. 403-417, 2011.

[29] Q. Din and M. Hussain, "Controlling chaos and NeimarkSacker bifurcation in a host-parasitoid model," Asian Journal of Control, vol. 21, no. 3, pp. 1202-1215, 2019.

[30] Q. Din, "Qualitative analysis and chaos control in a densitydependent host-parasitoid system," International Journal of Dynamics and Control, vol. 6, no. 2, pp. 778-798, 2018.

[31] Y.-h. Zhang, W. Zhou, T. Chu, Y.-d. Chu, and J.-n. Yu, "Complex dynamics analysis for a two-stage Cournot duopoly game of semi-collusion in production," Nonlinear Dynamics, vol. 91, no. 2, pp. 819-835, 2018.

[32] J. Zhou, W. Zhou, T. Chu, Y.-x. Chang, and M.-j. Huang, "Bifurcation, intermittent chaos and multi-stability in a twostage Cournot game with R\&D spillover and product differentiation," Applied Mathematics and Computation, vol. 341, pp. 358-378, 2019.

[33] Y. X. Cao, W. Zhou, T. Chu, and Y. X. Chang, "Global dynamics and synchronization in a duopoly game with bounded rationality and consumer surplus," International Journal of Bifurcation and Chaos, vol. 29, no. 11, Article ID 1930031, 2019.

[34] W. Zhou and X. X. Wang, "On the stability and multistability in a duopoly game with R\&D spillover and price competition," Discrete Dynamics in Nature and Society, vol. 2019, Article ID 2369898, 20 pages, 2019. 